\title{
Review: cognitive and behaviour therapies are effective for chronic pain
}

Sources of funding: in part, NHSE Northern and Yorkshire $R$ and $D$ and NHS Health

Technology Programme.

For correspondence: $D r$

$S$ Morley, Division of

Psychiatry and

Behavioural Sciences,

School of Medicine,

University of Leeds,

Leeds LS2 9JT, UK.

Fax $+44(0) 113243$

3719 .
Morley S, Eccleston C, Williams A. Systematic review and meta-analysis of randomized controlled trials of cognitive
behaviour therapy and behaviour therapy for chronic pain in adults, excluding headache. Pain 1999 Mar;80:1-13.

QUESTIONS: In patients with chronic pain, excluding headache, how effective is psychological treatment (including cognitive behavioural therapy [CBT], behavioural therapy, and biofeedback), and is it more effective than alternative active treatments?
Psychological treatment $v$ waiting list control (WLC) and active treatment control (ATC)

\begin{tabular}{lll}
\multirow{2}{*}{ Domain } & Treatment $v$ WLC & Treatment $v$ ATC \\
\cline { 2 - 3 } Pain experience & Mean effect size $(95 \%$ Cl) & Mean effect size (CI) \\
\hline Mood or affect (depression) & $0.40(0.22$ to 0.58$)$ & $0.29(0.11$ to 0.46$)$ \\
\hline Mood or affect (other) & $0.36(0.13$ to 0.59$)$ & $-0.14(-0.32 \text { to } 0.04)^{\star}$ \\
\hline Cognitive coping and appraisal (negative) & $0.50(0.27$ to 0.73$)$ & $0.17(-0.08 \text { to } 0.42)^{*}$ \\
\hline Cognitive coping and appraisal (positive) & $0.53(0.28$ to 0.78$)$ & $0.40(0.21$ to 0.62$)$ \\
\hline Behavioural expression of pain & $0.50(0.22$ to 0.78$)$ & $0.27(0.08$ to 0.47$)$ \\
\hline Increased behaviour activity & $0.46(0.25$ to 0.72$)$ & - \\
\hline Social role functioning & $0.60(0.44$ to 0.76$)$ & $0.17(-0.08 \text { to } 0.34)^{*}$ \\
\hline
\end{tabular}

*Not significant.

\section{COMMENTARY}

The limited success of conventional medical approaches to the treatment of chronic pain has in part facilitated the development of psychological treatment methods-predominantly cognitive behavioural in nature; patients entering such programmes will usually have obtained only limited relief from medication alone and often have depression, along with cognitive, behavioural, and social dysfunction. Thus elimination of pain, or even the reduction of pain intensity per se, are seldom core aims of pain management programmes (CBT or otherwise), and this accounts for the wider than usual domain of outcomes identified in the review by Morley et al.

This systematic review provides a thorough and valuable research synthesis in a field which is underdeveloped compared with mainstream psychotherapy outcome research. In this respect the meta-analysis might be considered less a review of formal efficacy research (ie, testing an exact model of treatment) and closer to a review of effectiveness studies (ie, concerned more with real world clinical effects of a broad CBT based approach).

With this in mind, this review shows that CBT based approaches (including behaviour therapy) are more clinically effective than simply waiting for further treatment for improving outcomes in patients with predominantly chronic low back pain and arthritis. They also show superiority-in some outcome domains - to the mixed bag of alternative treatments (eg, symptom monitoring, bibliotherapy, physiotherapy) in the studies reviewed.

The multicomponent nature of CBT approaches means that most studies evaluate the effect of a treatment package. The specificity of the effects of different treatment programme components in relation to different patient characteristics, problem domains, and outcomes, is an area of research in chronic pain management where limited progress has yet been made. Clinicians need to be mindful of this when considering delivering cut down versions of the multicomponent CBT approach where fewer components could simply mean lower effectiveness.

The fact that $76 \%$ of treatments were delivered in groups contrasts with most CBT research in psychiatric services where individual treatments have more commonly been evaluated. This may not only have economic significance, group therapy being cheaper to deliver, but must also be appreciated by clinicians wishing to work individually with pain patients. The role of group processes such as peer support remains as yet unknown as a determinant of chronic pain treatment outcome within the CBT framework.

Phil Richardson, $\mathrm{PhD}$ Tavistock and Portman NHS Trust London, UK

\section{Data sources}

Studies were identified by searching 4 databases and scanning reference lists.

\section{Study selection}

Selected studies were randomised controlled trials of cognitive behavioural treatments including behaviour therapy in adults who presented with chronic pain. Studies were excluded if they did not provide data for computing effect sizes.

\section{Data extraction}

Treatments were categorised into 3 primary types: biofeedback and relaxation, behaviour therapy, and CBT. Control groups were categorised as waiting list control and alternative treatment control. Because the studies had multiple measures, measurement domains were created: pain experience, mood or affect, cognitive coping and appraisal, pain behaviour, biology or physical fitness, social role functioning, use of healthcare system, and miscellaneous.

\section{Main results}

25 trials (1672 patients, mean age 58 y, $62 \%$ women) were included. Treatment was superior to waiting list control with significant effect sizes on all domains of measurement (table). The median effect size across all domains was 0.5 . Among the 3 treatment subtypes, CBT was more efficacious than waiting list control in all but 1 domain. When compared with alternative treatment control, effect sizes were significant for treatment for the domains of pain experience, cognitive coping and appraisal (positive coping), and reducing expression of pain (table). When treatment subtypes were analysed, the results were similar to those of the waiting list control comparison: CBT showed the greatest efficacy.

\section{Conclusions}

In patients with chronic pain, psychological treatment (including cognitive behaviour therapy, behaviour therapy, and biofeedback), is more effective than waiting list control conditions. Compared with alternative active treatments, psychological treatments are superior for such outcomes as pain experience, positive coping, and behavioural expression of pain. 